\title{
Sparse hypergraphs with low independence number
}

\author{
Jeff Cooper * Dhruv Mubayi ${ }^{\dagger}$
}

October 30, 2018

\begin{abstract}
Let $K_{4}^{(3)}$ denote the complete 3-uniform hypergraph on 4 vertices. Ajtai, Erdős, Komlós, and Szemerédi (1981) asked if there is a function $\omega(d) \rightarrow \infty$ such that every 3 -uniform, $K_{4}^{(3)}$-free hypergraph $H$ with $N$ vertices and average degree $d$ has independence number at least $\frac{N}{d^{1 / 2}} \omega(d)$. We answer this question by constructing a 3 -uniform, $K_{4}^{(3)}$-free hypergraph with independence number at most $2 \frac{N}{d^{1 / 2}}$. We also provide counterexamples to several related conjectures and improve the lower bound of some hypergraph Ramsey numbers.
\end{abstract}

\section{Introduction}

A $k$-uniform hypergraph $H$ is a pair $H=(V, E)$, where $V$ is the vertex set and $E \subset\left(\begin{array}{l}V \\ k\end{array}\right)$ is the edge set. We refer to the edge set of the hypergraph by $H$ and the vertex set by $V(H)$. The degree of a vertex in $V(H)$ is the number of edges containing that vertex. An independent set in a hypergraph is a subset of $V(H)$ which contains no edge of $H$. The independence number of $H$, denoted $\alpha(H)$, is the maximum size of an independent set in $H$. Turán [18] showed that $\alpha(G) \geq \frac{N}{d+1}$ for any graph $G$ with $N$ vertices and average degree $d$. Spencer [17] extended Turán's result to hypergraphs by showing that for all $k \geq 1$ there is a $c_{k}$ so that every $(k+1)$-uniform hypergraph $H$ with average degree $d$ satisfies $\alpha(H) \geq c_{k} \frac{N}{d^{1 / k}}$.

When $G$ is a graph, Turán's bound can be improved if $G$ is forbidden from containing a fixed subgraph. Ajtai, Komlós, and Szemerédi [3] showed that if $G$ is triangle-free,

*Department of Mathematics, Statistics, and Computer Science, University of Illinois at Chicago, Chicago, IL 60607, USA; email: jcoope8@uic.edu

${ }^{\dagger}$ Department of Mathematics, Statistics, and Computer Science, University of Illinois at Chicago, Chicago IL 60607, USA; research supported in part by NSF grant DMS-1300138; email: mubayi@uic.edu 
then

$$
\alpha(G) \geq \frac{1}{100} \frac{N}{d} \log d
$$

Ajtai, Erdős, Komlós, and Szemerédi [1] subsequently showed that if $t \geq 4$ and $G$ is $K_{t}$-free, then $\alpha(G) \geq c_{t} \frac{N}{d} \log \log d$.

Let $H$ be a $(k+1)$-uniform hypergraph with $N$ vertices and average degree $d$. Ajtai, Komlós, Pintz, Spencer, and Szemerédi [2] showed that there exists a positive constant $c_{k}$ such that if $H$ contains no 2,3 , or 4 cycles, then

$$
\alpha(H) \geq c_{k} \frac{N}{d^{1 / k}} \log ^{1 / k} d .
$$

Applications of (2) have been found in number theory [3], discrete geometry [12], coding theory [14], and Ramsey theory [4]. Ajtai, Erdős, Komlós and Szemerédi asked if, like in the graph case, (2) could also be extended to other families of hypergraphs.

Question 1 (Ajtai-Erdős-Komlós-Szemerédi [1]). Is there a function $\omega(d) \rightarrow \infty$ such that if a 3-uniform hypergraph $H$ contains no $K_{4}^{(3)}$ (or even $\left.K_{4}^{-(3)}\right)$, then $\alpha(H) \geq$ $\frac{N}{d^{1 / 2}} \omega(d)$ ?

We construct hypergraphs which negatively answer this question, even in the $K_{4}^{-(3)}$ case. The construction is presented in Section 2, In Section 3, we generalize this construction to $k$-uniform hypergraphs and disprove several conjectures related to Question 11. We also discuss an application to hypergraph Ramsey numbers.

\section{3-uniform construction}

In this section, we answer Question 1 by constructing a $K_{4}^{-(3)}$-free, 3-uniform hypergraph $H$ with independence number at most $2 N / d^{1 / 2}$. The hypergraph $H$ is constructed from the complete bipartite graph $K_{n, n}$ with vertex classes $[n]$ and $[n]$. The vertices of $H$ correspond to edges in the graph, while the edges of $H$ correspond to 3-edge paths which open in the increasing direction:

$$
\begin{aligned}
& V(H)=[n] \times[n] \\
& E(H)=\{\{a b, a c, d b \in[n] \times[n]: c>b, d>a\} .
\end{aligned}
$$

$H$ is clearly 3-uniform, contains $N=n^{2}$ vertices, and has average degree $d=3(n-1)^{2} / 4$. For $v \in V(H)$, consider the link graph $L_{v}=\{u w: u v w \in E(H)\}$. The components of $L_{v}$ are either stars (when $v$ is in the role of $a c$ ) or a bipartite graph (when $v$ is in the role of $a b) . K_{4}^{-(3)}$, on the other hand, contains a vertex whose link graph contains a triangle, so $H$ must be $K_{4}^{-(3)}$-free. 
Let $S \subset V(H)$. If $|S| \geq 2 n$, then the edges in $K_{n, n}$ corresponding to the vertices in $S$ contain a cycle on at least four vertices. The smallest vertex on this cycle is contained in a 3-edge path which opens in the increasing direction, and this path corresponds to an edge in $H$. Therefore, $\alpha(H)<2 n<2 N / d^{1 / 2}$.

\section{$3 \quad$ Related problems and conjectures}

\subsection{Ramsey numbers for 3-uniform tight paths}

Our construction also provides the correct order of magnitude for some new 3-uniform Ramsey numbers. Let $F$ be a 3-uniform hypergraph. Recall that the Ramsey number $r(F, t)$ is the smallest $n$ so that every red-blue coloring of the edges of $K_{n}^{(3)}$ contains a red $F$ or a blue complete $K_{t}^{(3)}$. Let $P_{s}$ denote the 3 -uniform hypergraph with vertex set $[s+2]$ and edge set $\{\{i, i+1, i+2\}: i \in[s]\} . P_{s}$ is called the 3 -uniform tight path. Results of Phelps and Rödl [16] imply that the Ramsey number of $P_{2}$ satisfies

$$
r\left(P_{2}, t\right)=\Theta\left(t^{2} / \log t\right) .
$$

It is easy to prove that for fixed $s$, we have $\operatorname{ex}\left(n, P_{s}\right)=O\left(n^{2}\right)$ and this immediately implies that

$$
r\left(P_{s}, t\right)=O\left(t^{2}\right) .
$$

Indeed, if we have a $P_{s}$-free 3-uniform hypergraph on $c_{s} n$ vertices $\left(c_{s}\right.$ large), then its average degree is at most $c_{s}^{\prime} n$, so it has an independent set of size at least $t=c_{s}^{\prime \prime} n^{1 / 2}$.

We now show that the construction in Section 2 contains no $P_{4}$, which improves the lower bound of $r\left(P_{s}, t\right)$ for $s \geq 4$. The order of magnitude of $r\left(P_{3}, t\right)$ remains open.

Theorem 2. Fix $s \geq 4$. Then $r\left(P_{s}, t\right)=\Theta\left(t^{2}\right)$.

Proof. We only need to prove the lower bound, which follows by observing that the hypergraph $H$ in Section 2 contains no $P_{4}$. Recall that every link graph of $H$ has one component that is a complete bipartite graph and all of its other components are stars; further, the pairs of vertices which form edges in the bipartite component appear in exactly one edge of $H$. On the other hand, the link graph of each of the degree 3 vertices in $P_{4}$ contains a 3 -edge path and one of the pairs of vertices which form an edge in this path is contained in two edges of $P_{4}$. 


\subsection{Generalization to $k$-uniform hypergraphs}

The construction in Section 2 starts with a bipartite graph and builds a hypergraph whose edges correspond to 3-edge paths in the graph. In this section, we generalize this method by starting with a multipartite hypergraph and building a new hypergraph whose edges correspond to some fixed hypergraph. The resulting hypergraphs provide counterexamples to various conjectures concerning $k$-uniform hypergraphs.

\subsubsection{Chromatic number of $k$-uniform hypergraphs}

A proper coloring of a hypergraph $H$ is a partition of $V(H)$ into independent sets. The chromatic number of $H$, denoted $\chi(H)$, is the minimum number of parts needed in a proper coloring of $H$. Erdős and Lovász [9] showed that every $(k+1)$-uniform hypergraph with maximum degree $\Delta$ has $\chi(H) \leq c_{k} \Delta^{1 / k}$. Strengthening (2), Frieze and the second author [11] showed that every $(k+1)$-uniform linear hypergraph with maximum degree

$\Delta$ satisfies $\chi(H) \leq c_{k}^{\prime}\left(\frac{\Delta}{\log \Delta}\right)^{1 / k}$. In [10, 11], the same authors conjectured a stronger positive answer to the question of Ajtai, Erdős, Komlós, and Szemerédi.

Conjecture 3 (Frieze-Mubayi [10, 11]). If $F$ is a $(k+1)$-uniform hypergraph and $H$ is an $F$-free $(k+1)$-uniform hypergraph with maximum degree $\Delta$, then $\chi(H) \leq$ $c_{F}(\Delta / \log \Delta)^{1 / k}$.

Let $T_{k}$ be the $k$-uniform hypergraph with $k+1$ edges $e_{1}, \ldots, e_{k}, f$ where for all $i \neq j$ we have $e_{i} \cap e_{j}=S$ and $f \supset e_{i}-S$ for some $S$ with $|S|=k-1$. In other words, $k$ edges share the same set of $k-1$ points and the last edge contains the remaining vertex from each of the $k$ edges. A $k$-uniform hypergraph has independent neighborhoods if it contains no copy of $T_{k}$. Bohman, Frieze, and the second author [5] conjectured a weaker version of Conjecture 3: if $H$ is a 3-uniform hypergraph with maximum degree $\Delta$ and independent neighborhoods, then $\chi(H)=o\left(\Delta^{1 / 2}\right)$.

The construction in Section 2 shows that both of these conjectures are false for 3uniform hypergraphs. We now generalize that construction to disprove these conjectures for $k$-uniform hypergraphs.

\subsubsection{Construction from positive strong $k$-simplices}

Fix $k \geq 2$. A $k$-simplex is a collection of $k+1$ sets with empty intersection, every $k$ of which have nonempty intersection. A strong $k$-simplex $S_{k}$, introduced in [15], is the $k$-uniform hypergraph with vertex set $\left\{v_{1}, v_{1}^{\prime}, \ldots, v_{k}, v_{k}^{\prime}\right\}$ and edge set $\left\{e, e_{1}, \ldots, e_{k}\right\}$ 
where $e=\left\{v_{1}, \ldots, v_{k}\right\}$ and $e_{i}=e \cup\left\{v_{i}^{\prime}\right\}-v_{i}$ ( $e$ is called the central edge). Given disjoint sets $X_{1}, \ldots, X_{k}$ with each $X_{i} \cong[n]$, a positive strong $k$-simplex $S_{k}^{+}$is a $k$-partite strong simplex satisfying $v_{i}, v_{i}^{\prime} \in X_{i}$ and $v_{i}^{\prime}>v_{i}$ for each $i=1, \ldots, k$.

Let $X_{1}, \ldots, X_{k}$ be disjoint sets each isomorphic to $[n]$. Define the $(k+1)$-uniform hypergraph $H_{k}$ with vertex set $X_{1} \times \cdots \times X_{k}$ and edge set

$$
H_{k}=\left\{A \subset X_{1} \times \cdots \times X_{k}: A \cong S_{k}^{+}\right\}
$$

For example, $\mathrm{H}_{2}$ corresponds to the construction in Section 2 ,

Fix a $k$-uniform hypergraph $F_{k}$. The Zarankiewicz number $z\left(n, F_{k}\right)$ is the maximum number of edges in a $k$-partite $k$-uniform hypergraph with parts of size $n$ that contains no copy of $F_{k}$. Since copies of $S_{k}^{+}$correspond to edges of $H_{k}$,

$$
\alpha\left(H_{k}\right) \leq z\left(n, S_{k}^{+}\right)
$$

We may thus use the following lemma below to bound $\alpha\left(H_{k}\right)$.

Lemma 4. Fix $k \geq 2$. Then $z\left(n, S_{k}^{+}\right) \leq 2 k n^{k-1}$.

Proof. We proceed by induction on $k$. The base case $k=2$ follows from Section 2 , For the induction step, suppose we are given a $k$-partite $H \subset X_{1} \times \cdots \times X_{k}$ with $|H|>2 k n^{k-1}$, where each $X_{i} \cong[n]$. For a vertex $v$ in a $k$-uniform hypergraph $H$, define its link to be the $(k-1)$-uniform hypergraph $L_{v}=\{S \subset V(H): v \notin S, S \cup\{v\} \in H\}$. For a set of vertices $T$, let $d_{H}(T)$ denote the number of edges containing $T$.

For each $v \in X_{1}$, let $L_{v}$ be the link $(k-1)$-uniform hypergraph of $v$. Let $A_{v} \subset L_{v}$ comprise those $(k-1)$-sets $T$ with $d_{H}(T)=1$ and $B_{v}=L_{v}-A_{v}$.

Let $B_{v}^{+}$be the set of all $S \in B_{v}$ such that there exists $v^{\prime}>v$ with $S \in L_{v^{\prime}}$. We will find $x \in X_{1}$ with $\left|B_{x}^{+}\right|>2(k-1) n^{k-2}$ and then apply induction. Now

$$
\sum_{v \in X_{1}}\left|B_{v}^{+}\right|=\sum_{\substack{S \in X_{2} \times \cdots \times X_{k}: \\ d_{H}(S) \geq 2}}\left(d_{H}(S)-1\right) \geq \sum_{\substack{S \in X_{2} \times \cdots \times X_{k}: \\ d_{H}(S) \geq 2}} d_{H}(S)-n^{k-1}=\sum_{v \in X_{1}}\left|B_{v}\right|-n^{k-1} .
$$

Thus

$$
\begin{aligned}
2 k n^{k-1}<|H|=\sum_{v \in X_{1}}\left|L_{v}\right|=\sum_{v \in X_{1}}\left|A_{v}\right|+\sum_{v \in X_{1}}\left|B_{v}\right| & \leq n^{k-1}+\sum_{v \in X_{1}}\left|B_{v}\right| \\
& \leq 2 n^{k-1}+\sum_{v \in X_{1}}\left|B_{v}^{+}\right| .
\end{aligned}
$$

Consequently, there exists $x \in X_{1}$ with $\left|B_{x}^{+}\right|>2(k-1) n^{k-2}$. Apply induction to $B_{x}$ to obtain a copy of $S_{k-1}^{+}$in $X_{2} \times \cdots \times X_{k}$. To form $S_{k}^{+}$, begin by enlarging each edge 
of $S_{k-1}^{+}$with $x$. Add another edge by enlarging the central edge $e$ by some other vertex $y \in X_{1}$ with $y>x$. Note that $y$ exists since $e \in B_{x}^{+}$and $d_{H}(e)>1$. We have thus obtained a copy of $S_{k}^{+}$, where $e \cup\{x\}$ is the central edge.

Notice $H_{k}$ has $N=n^{k}$ vertices and maximum degree $\Delta \leq(k+1) n^{k}$. By Lemma 4,

$$
\alpha\left(H_{k}\right) \leq 2 k n^{k-1}=2 k(k+1)^{1 / k} \frac{n^{k}}{\left((k+1) n^{k}\right)^{1 / k}} \leq 2 k(k+1)^{1 / k} \frac{N}{\Delta^{1 / k}},
$$

and

$$
\chi\left(H_{k}\right) \geq \frac{\Delta^{1 / k}}{2 k(k+1)^{1 / k}} .
$$

Recall that $T_{k+1}$ is the $(k+1)$-uniform hypergraph with $k+2$ edges $e_{1}, \ldots, e_{k+1}, f$ where for all $i \neq j, e_{i} \cap e_{j}=S$ and $f \supset e_{i}-S$ for some $S$ with $|S|=k$. Suppose $S_{k, 1}^{+}, \ldots, S_{k, k+1}^{+}$satisfy $S_{k, i}^{+} \cap S_{k, j}^{+}=S$, for $i \neq j$ and $|S|=k$. Since each strong $k$-simplex is positive, they must share a single central edge. Thus the edges in $\left(S_{k, 1}^{+} \cup \cdots \cup S_{k, k+1}^{+}\right)-S$ share a single vertex and so do not form a positive strong $k$-simplex. Therefore $H_{k}$ does not contain any copy of $T_{k+1}$, disproving Conjecture 3 and the weaker conjecture of [5].

\subsection{3 c-sparse hypergraphs}

A hypergraph is c-sparse if every vertex subset $S$ spans at most $c|S|^{2}$ edges. By Spencer's extension of Turán's bound, every c-sparse hypergraph $H$ with $N$ vertices satisfies $\alpha(H) \geq c_{k}^{\prime} \sqrt{N}$. Phelps and Rödl [16] improved this to $\alpha(H) \geq c_{k}^{\prime} \sqrt{N \log N}$ for linear 3-uniform hypergraphs. In 1986, de Caen (see [7]) conjectured that a similar improvement holds even for $c$-sparse hypergraphs (observe that linear implies $\frac{1}{2}$-sparse).

Conjecture 5 (De Caen [7]). For every positive $c$, there is a function $\omega(N) \rightarrow \infty$ such that every c-sparse 3-uniform hypergraph $H$ with $N$ vertices satisfies $\alpha(H) \geq \omega(N) \sqrt{N}$.

Recently, Kostochka, the second author, and Verstraëte [13] posed a stronger version of de Caen's conjecture: for every positive $c$, there is a function $\omega(N) \rightarrow \infty$ such that every $c$-sparse 3 -uniform hypergraph $H$ with $N$ vertices and average degree $d$ satisfies $\alpha(H) \geq \omega(N) \frac{N}{d^{1 / 2}}$.

Observe that the construction in Section 2 is 1-sparse, so $S_{2}$ immediately provides a counterexample to Conjecture 5 and the conjecture of [13]. However, for $k \geq 3, H_{k}$ is not $c$-sparse for any constant $c$, so one may ask whether or not for $k \geq 3$ and every positive $c$ there is a function $\omega(N) \rightarrow \infty$ such that every $c$-sparse $(k+1)$-uniform hypergraph $H$ with $N$ vertices satisfies $\alpha(H) \geq \omega(N) N^{1 / k}$. The next section provides a counterexample to this generalization of de Caen's conjecture. 


\subsubsection{Construction from special $k$-clusters}

A $k$-cluster, introduced in [15], is a collection of $k+1$ sets with empty intersection whose union has size at most $2 k$. The family of special $k$-clusters $\mathcal{D}_{k}$ is the $k$-uniform hypergraph family that is defined inductively as follows: $\mathcal{D}_{2}=\left\{D_{2}\right\}$, where $D_{2}$ is the path with three edges. For $k \geq 3, \mathcal{D}_{k}$ is the family of $k$-uniform hypergraphs which can be constructed as follows: begin with any $D_{k-1} \in \mathcal{D}_{k-1}$, which is assumed inductively to have $2(k-1)$ vertices and two disjoint edges $a$ and $b$. Then $D_{k}$ is a member of $\mathcal{D}_{k}$ if it can be formed by adding two new vertices $x, y$ to $D_{k-1}$, enlarging all edges of $D_{k-1}$ by including $x$, and enlarging $a$ by including $y$. Thus $D_{k}$ has $2 k$ vertices and $k+1$ edges, two of which are disjoint. We will use $D_{k}$ to denote an arbitrarily chosen member of $\mathcal{D}_{k}$.

Following the construction from Section 3.2.2, define the $(k+1)$-uniform hypergraph $J_{k}$ with vertex set $X_{1} \times \cdots \times X_{k}$ and edge set

$$
J_{k}=\left\{A \subset X_{1} \times \cdots \times X_{k}: A \cong D_{k}\right\} .
$$

Lemma 6. Fix $k \geq 2$ and $D_{k} \in \mathcal{D}_{k}$. Then $z\left(n, D_{k}\right) \leq k n^{k-1}$.

Proof. We proceed by induction on $k$. For the base case $k=2$, observe that $D_{2}$ is the path with 3 edges. If $H$ is a bipartite graph with more than $2 n$ edges, then $H$ contains a cycle with at least four edges, which contains a copy of $D_{2}$. For the induction step, suppose we are given $k$-partite $H$ with $|H|>k n^{k-1}$ with parts $X_{1}, \ldots, X_{k}$ each of size $n$. For each $v \in X_{1}$, define $L_{v}, A_{v}$, and $B_{v}$ as in the proof of Lemma 4 . Then

$$
k n^{k-1}<|H|=\sum_{v \in X_{1}}\left|L_{v}\right|=\sum_{v \in X_{1}}\left|A_{v}\right|+\sum_{v \in X_{1}}\left|B_{v}\right| \leq n^{k-1}+\sum_{v \in X_{1}}\left|B_{v}\right| .
$$

Consequently, there exists $x \in X_{1}$ with $\left|B_{x}\right|>(k-1) n^{k-2}$. Let $D_{k-1}$ be the member of $\mathcal{D}_{k-1}$ that gives rise to $D_{k}$ in the inductive construction of $D_{k}$. Apply induction to $B_{x}$ to obtain a copy of $D_{k-1}$ in $B_{x}$. To form $D_{k}$, begin by enlarging each edge of $D_{k-1}$ with $x$. Add another edge by enlarging one of the two disjoint edges $a, b$ of $D_{k-1}$ (say $a$ ) by some other vertex $y \in X_{1}$. Note that $y$ exists since $a \in B_{x}$. We have thus obtained a copy of $D_{k}$, where $a \cup\{y\}$ and $b \cup\{x\}$ are the disjoint edges.

By Lemma 6, $\alpha\left(J_{k}\right) \leq k n^{k-1}$, so it suffices to show that $J_{k}$ is $2^{2 k^{2}-2 k-1}$-sparse. Let $S \subset V\left(J_{k}\right)$. The vertex set of a copy of $D_{k}$ is determined by the two disjoint edges in $D_{k}$. There are at most $\left(\begin{array}{c}2 k \\ k\end{array}\right)^{k-1}$ possibilities for the remaining $k-1$ edges. Therefore we may associate every pair of vertices in $S$ to at most $\left(\begin{array}{c}2 k \\ k\end{array}\right)^{k-1}$ edges in the subgraph induced by $S$. Since every edge corresponds to at least one pair of vertices, the number 
of edges in $S$ is at most

$$
\left(\begin{array}{c}
2 k \\
k
\end{array}\right)^{k-1}\left(\begin{array}{c}
|S| \\
2
\end{array}\right)<2^{2 k^{2}-2 k-1}|S|^{2} .
$$

This disproves the generalization of de Caen's conjecture to $k$-uniform hypergraphs.

\section{Concluding remarks}

- $H_{k}$ is a counterexample to Conjecture 3 with $N$ vertices and maximum degree $\Theta(N)$. Sparser counterexamples with $f N$ vertices and maximum degree $N$ can be constructed by taking the disjoint union of $f$ copies of $H_{k}$.

- Benny Sudakov suggested the following generalization of $H_{2}$ to $(k+1)$-uniform hypergraphs, which provides a denser counterexample to Conjecture 3 for $k \geq 3$. Let $G$ be the $(k+1)$-uniform hypergraph with vertex set $[n] \times[n]$ and edge set

$$
\left\{\left(x_{1}, y_{1}\right),\left(x_{1}, y_{2}\right),\left(x_{2}, y_{2}\right), \ldots,\left(x_{k}, y_{2}\right): y_{2}<y_{1}, x_{i}<x_{i+1} \text { for } i \in[k-1]\right\} \text {. }
$$

In other words, each edge corresponds to an $L$ with $k$ points on its base. It is not hard to see that $G$ has maximum degree $\Theta\left(n^{k}\right)$, independence number $\Theta(n)$, and contains no copy of $T_{k+1}$.

- For $1<r<k+1$, say that a $(k+1)$-uniform hypergraph is $(c, r)$-sparse if every vertex subset $S$ spans at most $c|S|^{r}$ edges. A partial Steiner $(k+1, k)$-system is a $(k+1)$-uniform hypergraph with every $k$ vertices in at most one edge. Such a system has average degree at most $n^{k-1}$ and, by [13], has independence number at least $c^{\prime}(n \log n)^{1 / k}$ for some positive $c^{\prime}$. This result cannot be extended to the larger class of $(c, k)$-sparse $(k+1)$-uniform hypergraphs, as shown by the following $(c, 3)$-sparse 4-uniform hypergraphs with independence number $O\left(n^{1 / 3}\right)$.

Let $F$ be the set of 3-partite 3-uniform hypergraphs with four edges such that one of the edges is contained in the union of the other three. Then it is an easy exercise to show (by induction on $n$ for example) that $z(n, F)=O(n)$, so our general construction provides a 4-uniform, $(c, 3)$-sparse hypergraph $H(F)$ on $n^{3}$ vertices with $\alpha(H(F))=O(n)$ (for $(c, 3)$-sparse, use the argument in Section 3.2.4).

We remark that, in addition, $H(F)$ contains no $K_{163}^{(4)}$ for the vertex set of a copy of $K_{163}^{(4)}$ would correspond to a set of $163=1+3 !(4-1)^{3} 3$-uniform edges, and by the Erdős-Rado sunflower lemma, these edges would contain a sunflower $C$ of size 4. But the 4 vertices in $H(F)$ corresponding to the edges of $C$ cannot form an edge in $H(F)$ since not one of them is contained in the union of the other three. 
- Define the 3-uniform hypergraphs $F_{5}=\{a b c, a b d, c d e\}$ and $C_{3}=\{a b c, c d e, e f a\}$. The authors [6] recently answered Question 1 positively if, in addition to $K_{4}^{-(3)}$, $F_{5}$ and $C_{3}$ are also forbidden. It would be interesting to answer Question 1 if only $K_{4}^{-(3)}$ and $C_{3}$ are forbidden.

\section{Acknowledgments}

We would like to thank the referees for carefully reading our manuscript and providing thoughtful feedback.

\section{References}

[1] M. Ajtai, P. Erdős, J. Komlós, and E. Szemerédi, On Turán's theorem for sparse graphs, Combinatorica 1 (1981), no. 4, 313-317. MR 647980 (83d:05052)

[2] M. Ajtai, J. Komlós, J. Pintz, J. Spencer, and E. Szemerédi, Extremal uncrowded hypergraphs, J. Combin. Theory Ser. A 32 (1982), no. 3, 321-335. MR 657047 (83i:05056)

[3] M. Ajtai, J. Komlós, and E. Szemerédi, A dense infinite Sidon sequence, European J. Combin. 2 (1981), no. 1, 1-11. MR 611925 (83f:10056)

[4] N. Alon, T. Jiang, Z. Miller, and D. Pritikin, Properly colored subgraphs and rainbow subgraphs in edge-colorings with local constraints, Random Structures Algorithms 23 (2003), no. 4, 409-433. MR 2016871 (2004i:05106)

[5] T. Bohman, A. Frieze, and D. Mubayi, Coloring $\mathcal{H}$-free hypergraphs, Random Structures Algorithms 36 (2010), no. 1, 11-25. MR 2591044 (2011e:05081)

[6] J. Cooper and D. Mubayi, List coloring triangle-free hypergraphs, (submitted) (2013).

[7] D. de Caen, The current status of Turán's problem on hypergraphs, Extremal problems for finite sets (Visegrád, 1991), Bolyai Soc. Math. Stud., vol. 3, János Bolyai Math. Soc., Budapest, 1994, pp. 187-197. MR 1319162 (95m:05127)

[8] R. A. Duke, H. Lefmann, and V. Rödl, On uncrowded hypergraphs, Proceedings of the Sixth International Seminar on Random Graphs and Probabilistic Methods in Combinatorics and Computer Science, "Random Graphs '93" (Poznań, 1993), vol. 6, 1995, pp. 209-212. MR 1370956 (96h:05146) 
[9] P. Erdős and L. Lovász, Problems and results on 3-chromatic hypergraphs and some related questions, Infinite and finite sets (Colloq., Keszthely, 1973; dedicated to P. Erdős on his 60th birthday), Vol. II, North-Holland, Amsterdam, 1975, pp. 609627. Colloq. Math. Soc. János Bolyai, Vol. 10. MR 0382050 (52 \#2938)

[10] A. Frieze and D. Mubayi, On the chromatic number of simple triangle-free triple systems, Electron. J. Combin. 15 (2008), no. 1, Research Paper 121, 27. MR 2443136 (2009j:05170)

[11] _ Coloring simple hypergraphs, Journal of Combinatorial Theory. Series B (to appear).

[12] J. Komlós, J. Pintz, and E. Szemerédi, A lower bound for Heilbronn's problem, J. London Math. Soc. (2) 25 (1982), no. 1, 13-24. MR 645860 (83i:10042)

[13] A. Kostochka, D. Mubayi, and J. Verstraete, On independent sets in hypergraphs, Random Structures Algorithms (to appear).

[14] H. Lefmann, Sparse parity-check matrices over GF(q), Combin. Probab. Comput. 14 (2005), no. 1-2, 147-169. MR 2128087 (2006b:94064)

[15] D. Mubayi, An intersection theorem for four sets, Adv. Math. 215 (2007), no. 2, 601-615. MR 2355601 (2008m:05295)

[16] K. T. Phelps and V. Rödl, Steiner triple systems with minimum independence number, Ars Combin. 21 (1986), 167-172. MR 846690 (87j:05038)

[17] J. Spencer, Turán's theorem for k-graphs, Discrete Math. 2 (1972), 183-186. MR 0297614 (45 \#6668)

[18] P. Turán, On an extremal problem in graph theory (in hungarian), Math. Fiz. Lapok 48 (1941), 436-452. 